\title{
Protective effect of extracorporeal membrane pulmonary oxygenation combined with cardiopulmonary resuscitation on post- resuscitation lung injury: a randomised controlled trial
}

Jiyang Ling

Beijing Tongren Hospital

Chunsheng Li ( $\nabla$ lcscyyy@163.com)

Capital Medical University Affiliated Beijing Friendship Hospital https://orcid.org/0000-0002-31614457

\section{Yun Zhang}

Beijing Tongren Hospital

Xiaoli Yuan

Beijing Tongren Hospital

Bo Liu

Beijing Chaoyang Hospital

Yong Liang

Beijing Chaoyang Hospital

Qiang Zhang

Beijing Chaoyang Hospital

Original research

Keywords: Cardiac arrest, swine, alveolar surface-active protein, oxidative stress injury, pulmonary oedema

Posted Date: June 26th, 2020

DOI: https://doi.org/10.21203/rs.3.rs-37919/v1

License: (c) (i) This work is licensed under a Creative Commons Attribution 4.0 International License. Read Full License 


\section{Abstract}

Background: This work examines the protective effect and mechanisms of early extracorporeal membrane oxygenation with cardiopulmonary resuscitation (CPR) on ventricular-fibrillation-induced postresuscitation lung injury in a swine cardiac-arrest model.

Methods: Sixteen male swine were randomised to conventional CPR (CCPR; $n=8 ; C C P R$ alone) and extracorporeal $\mathrm{CPR}(\mathrm{ECPR} ; \mathrm{n}=8$; extracorporeal membrane oxygenation with $\mathrm{CCPR})$, with restoration of spontaneous circulation for $6 \mathrm{~h}$ as an endpoint. Serological specimens were collected at baseline and restoration of spontaneous circulation for 1, 2, 4, and 6 h; lung tissue specimens were obtained postmortem. Between-group comparisons of recovery success rate, extravascular lung water, pulmonary vascular permeability index, electron microscopic features, and serum and tissue biomarkers (surfactant protein A, surfactant protein D, Clara cell protein 16 , superoxide dismutase, malondialdehyde, myeloperoxidase) were undertaken.

Results: The CCPR group had non-significantly lower 6-h survival rate $(p>0.05)$. Serum levels of surfactant protein $A$, surfactant protein $D$, Clara cell protein 16 , and malondialdehyde were significantly higher $(p<0.05$ ), whereas serum superoxide dismutase was significantly lower, in the CCPR than in the ECPR group $(p<0.01)$. Compared with the ECPR group, tissue surfactant protein $A$, surfactant protein $D$, and superoxide dismutase significantly decreased compared to the baseline, whereas malondialdehyde and myeloperoxidase significantly increased $(p<0.01)$ in the CCPR group. Extravascular lung water and pulmonary vascular permeability index were significantly higher in the CCPR after $6 \mathrm{~h}$ compared to the baseline values and the ECPR group $(p<0.01)$.

Conclusions: Electron microscopy revealed mostly vacuolated intracellular alveolar type II lamellar bodies and fuzzy lamellar structure and widening and blurring of the blood-gas barrier in the CCPR group in contrast to that in the ECPR group. ECPR was found to have protective pulmonary effects, possibly related to the regulation of alveolar surface-active proteins and mitigated oxidative stress response postresuscitation.

\section{Background}

Cardiac arrest (CA) is a critical condition that is a concern for healthcare workers worldwide.

Cardiopulmonary resuscitation (CPR) is widely used in CA of various etiologies, which can be cardiogenic (e.g. myocardial infarction, malignant ventricular arrhythmia, etc.) or non-cardiogenic (e.g. acute pulmonary embolism, severe infection, etc.) [1] although, the main pathogenic factors are still considered 
cardiogenic [2]. CPR has long been regarded as the only effective mechanism to improve the survival rate and prognosis following CA. However, combined with all aspects of in-hospital and out-hospital data, the CA-associated mortality rate has not significantly improved for a long time and remains very high $[2,3]$. Moreover, when the duration of CA exceeds $10 \mathrm{~min}$, the survival rate declines rapidly; if the duration of CA exceeds $20 \mathrm{~min}$, patients rarely survive [4].

Extracorporeal membrane oxygenation (ECMO) is a technology that relies on instrumentation to support the basic needs of the human body and has found widespread application in the treatment of various diseases. Since the 1990s, ECMO combined with CPR technology (extracorporeal cardiopulmonary resuscitation [ECPR]) has been increasingly applied in attempts to improve the success rate of recovery following a CA [5], and there are many reports of successful patient recovery through ECPR worldwide. Several studies have analysed and summarised relevant cases and comparatively studied ECPR and conventional CPR (CCPR) technology to suggest that ECPR is superior to CCPR [6-9]. However, the currently available studies are mostly retrospective studies or case analyses, and there is a lack of multicenter randomised controlled clinical trials that compare the protective effects of the two resuscitative methods on organs, especially because there are no reports of the changes of compression time and the effect of early oxygenated blood perfusion in relieving post-resuscitation lung injury in experimental animals (swine) [10-12].

The primary purpose of this experiment in an animal model of ventricular fibrillation-induced CA in swine was to study whether the early application of ECPR has advantages over CCPR in lung injury and to explore the protective mechanism of ECPR on the post-resuscitation pulmonary injury.

\section{Methods}

\section{Ethical approval}

This study was approved by the Capital Medical University Institutional Animal Care Committee and the Beijing Chao-Yang Hospital Affiliated to the Capital Medical University Animal Care and Use Committee.

\section{Animal preparation}

We used 40-day-old male long-white swines $(n=16$; weight $35.13 \pm 5.57 \mathrm{~kg})$ in this experiment. The experimental animals were randomly divided into the CCPR (CCPR after CA, $n=8$ ) and ECPR groups (ECPR after $\mathrm{CA}, \mathrm{n}=8$ ). All experimental animals were subjected to overnight fasting before the experiment, but without restricting water intake. At the beginning of the experiment, anaesthesia was induced by intramuscular injections of ketamine $(10 \mathrm{mg} / \mathrm{kg})$ and midazolam $(0.5 \mathrm{mg} / \mathrm{kg})$. When the swine entered a state of relative sedation, auricular venipuncture was undertaken to establish venous access, and propofol $(2.0 \mathrm{mg} / \mathrm{kg})$ was intravenously administered. Thereafter, the swine were quickly transferred to the operating table and placed supine; propofol $(9.0 \mathrm{mg} / \mathrm{kg} / \mathrm{h})$ and fentanyl $(1 \mu \mathrm{g} / \mathrm{kg} / \mathrm{h})$ were continuously infused intravenously to achieve the therapeutic effects of anesthesia and analgesia. Endotracheal intubation was carried out with a $6.5-\mathrm{mm}$ tracheal catheter after confirmation of deep 
anesthesia by examination of the corneal reflex. After ascertaining correct placement of the endotracheal tube, the animals were placed on mechanical ventilation (Servo 900 c; Siemens, Berlin, Germany) with volume-controlled ventilation (tidal volume $15.0 \mathrm{~mL} / \mathrm{kg}$, respiratory rate 12-20 cycles/min, and oxygen concentration 35\%). Three ECG monitoring lines and a peripheral blood oxygen saturation monitor were connected through the body surface to a multi-function monitor (bl-420 F Data Acquisition and Analysis System; Chengdu TME Technology Co., Ltd., Sichuan, China) for continuous monitoring of the ECG and peripheral oxygen saturation. The tracheal tube outlet was connected to an end-expiratory carbon dioxide monitor (CO2SMOplus monitor; Respironics Inc., Murrysville, PA), which continuously monitors and records end-expiratory $\mathrm{CO}_{2}$ data and adjusts ventilatory parameters accordingly to maintain endexpiratory $\mathrm{CO}_{2} \mathrm{pCO}_{2}$ at $35-45 \mathrm{mmHg}$.

Subsequently, a 5-Fr deep venous catheter was inserted into the right external jugular vein of the experimental animal, for intravenous infusion and fibrillation access. After percutaneous separation, the left femoral artery and left femoral vein were exposed and cut open respectively, and an arterial catheter (5 Fr; Terumo, Tokyo, Japan) and Swan-Ganz catheter (7 Fr; Edwards Life Sciences, Irvine, CA, USA) were respectively placed and connected to an HP monitor (M1165; Hewlett-Packard Co., Palo Alto, CA) to continuously monitor the heart rate (HR) and mean arterial pressure (MAP) through the hot dilution method to detect extravascular lung water (EVLW), the pulmonary vascular permeability index (PVPI), and other relevant data. A 16-Fr (Dragon Laifu Medical Products Co., Ltd., Changzhou, Jiangsu, China) intravenous catheter was inserted into the right atrium, and a percutaneous incision was made to expose the right femoral artery through which a 14-Fr arterial catheter was inserted into the ascending aorta. These two catheters were connected to the ECMO machine. All the abovementioned surgical procedures were conducted in accordance with the principles of aseptic operation, and all catheters were pretreated with heparin normal saline $(5.0 \mathrm{U} / \mathrm{mL})$. Intraoperatively, normal saline $(5.0 \mathrm{~mL} / \mathrm{kg} / \mathrm{h})$, and colloid $(5.0$ $\mathrm{mL} / \mathrm{kg} / \mathrm{h}$ ) were routinely administered to replace the body fluids lost during the operation.

\section{Preparation of ECMO}

In this study, the ECMO equipment was made up of arterial and venous catheters, the Levitronix Centrimag control system (Sarns Healthcare/3M, Ann Arbor, MI, USA), a centrifugal force pump (MAQUET Cardiopulmonary AG, Rastatt, Germany), a coated porous biofilm lung (MAQUET Holding B.V. and Co), and gas mixers (Thoratec Corporation, Pleasanton, CA, USA). The ECMO apparatus was connected to the swine through arteriovenous catheters, and the catheter and ECMO machine were prewashed with heparinised normal saline and filled with approximately $1000 \mathrm{~mL}$ heparinised colloid solution $(250 \mathrm{U} / \mathrm{kg})$ oxygenated up to $50 \%$ oxygen through the oxygenator. Moreover, the oxygenator was used to adjust the input ratio between oxygen and air, and a heater was used to ensure a constant temperature of $34^{\circ} \mathrm{C}$. However, until the ECMO machine was in operation, the colloidal fluid in the machine did not intermingle with the body fluids of the swine.

\section{Experimental procedure}


After the aforementioned preparation, the swine were placed under observation for $30 \mathrm{~min}$. Thereafter, the baseline parameters of the animals were recorded, and blood samples were collected. Care was taken to ensure the animals are in a relatively stable state before the onset of artificial ventricular fibrillation.

In this experiment, a medical programmed stimulator (GY-600A; Kaifeng Huanan Instrument Co., Kaifeng, Henan, China) was used to induce fibrillation (parameters: mode S1/S2 [300/200 ms], output voltage 40 V, 8:1 ratio, step-size -10 ms continuous program-controlled stimulation until ventricular fibrillation). The success of ventricular fibrillation was determined by the rapid decrease of arterial pressure and the occurrence of ventricular fibrillation on the monitoring ECG. The CCPR and ECPR groups were observed for 12 min, during which no treatment or intervention was undertaken in either group. Thereafter, CPR was conducted in both groups according to the 2015 guidelines [13] for CPR, but without any defibrillation treatment during this period. Fourteen min after the shock, the CCPR group was treated by the guidelines for CPR, whereas the ECPR group was treated with ECMO based on continuous CPR. The ECMO flow rate was set at $3-5 \mathrm{~L} / \mathrm{min}$, and the blood flow was maintained at $50-70 \%$ of the ejection fraction of the baseline state. The CCPR and ECPR groups received their first defibrillation at $18 \mathrm{~min}$ after the shock. The defibrillator was applied with a two-way wave of $4.0 \mathrm{~J} / \mathrm{kg}$ for defibrillation. During this period, epinephrine $(0.02 \mathrm{mg} / \mathrm{kg})$ was administered through the right external jugular vein cannula. Subsequent compressions, defibrillation, and medication use were in accordance with the 2015 guidelines for CPR [14].

The return of spontaneous circulation (ROSC) is applied when that the MAP or the systolic pressure is greater than 60 or $80 \mathrm{mmHg}$, respectively, for more than $20 \mathrm{~min}$ [15]. At this time, the CCPR group was connected to mechanical ventilation on a ventilator, the initial oxygen concentration was temporarily adjusted to $100 \%$, and the ventilatory parameters were adjusted according to peripheral oxygen saturation and $\mathrm{pCO}_{2}$ at the end of expiration. In the ECPR group, the oxygen concentration was adjusted according to the oxygenator parameters, and the common goal for both groups was the maintenance of end-expiratory $\mathrm{CO}_{2}$ at $35-45 \mathrm{mmHg}$. The endpoint observed in this experiment was the status at $6 \mathrm{~h}$ after the ROSC or the death of the experimental animal.

After $6 \mathrm{~h}$ of ROSC, the swine were injected intravenously with $60 \mathrm{mg}$ propofol before being administered $20 \mathrm{~mL} \mathrm{10 \%} \mathrm{potassium} \mathrm{chloride} \mathrm{and} \mathrm{sacrificed.}$

\section{Data collection and testing}

Haemodynamic parameters, including HR, MAP, and end-expiratory carbon monoxide, were monitored continuously. EVLW and PVPI were measured by the thermal dilution method at baseline in both groups; moreover, these were measured in the CCPR group at $6 \mathrm{~h}$ after ROSC, and in the ECPR group after the ECPR machine was stopped following 6-h ROSC. We used $5 \mathrm{~mL}$ ice brine three times for each measurement, and the mean value was noted. If the swine died prematurely, only the baseline values were measured. 
Arterial blood samples were collected through the left femoral artery catheter, with the first $5 \mathrm{~mL}$ of blood sample discarded to ensure the accuracy of the sample. An arterial blood gas analysis was undertaken and recorded by a blood gas analyser (GEM Premier 3000, Instrumentation Laboratory, Lexington, MA, USA).

Blood samples were collected at ROSC and at1, 2, 4, and $6 \mathrm{~h}$ after ROSC through the left femoral vein catheter, and the first $5 \mathrm{~mL}$ of blood was discarded before the corresponding samples were collected each time to ensure the accuracy of the relevant samples. Blood samples were centrifuged at $1000 \times g$, immediately at $4^{\circ} \mathrm{C}$ for $20 \mathrm{~min}$. The supernatant was stored at $-80^{\circ} \mathrm{C}$ until further analysis. The supernatant was tested by enzyme-linked immunosorbent assay (ELISA) on ELISA kits (Shanghai Sangon Biotechnology Company, Ltd., Shanghai, China) for pulmonary surfactant protein A (SP-A), pulmonary surfactant protein D (SP-D), Clara cell protein 16 (CC16), malondialdehyde (MDA), and superoxide dismutase (SOD).

Lung tissue samples (lower lobe of the right lung) were collected immediately after the swine were euthanised. The tissue was washed with phosphate-buffered saline (PBS) and placed in liquid nitrogen for rapid cold preservation. Testing was conducted at room temperature after the temperature returned to $2-8^{\circ} \mathrm{C}$, and the samples were mixed with PBS at a 1:9 weight volume treated with protease inhibitors and pressed by grass homogeniser fully ground on ice. Finally, the homogenate was centrifuged at $5000 \times g$ for $10 \mathrm{~min}$, and the supernatant was tested for myeloperoxidase (MPO), MDA, SOD, SP-A, and SP-D (Shanghai Sangon Biotechnology Company) by ELISA.

The lower lobe of the right lung was fixed with $3 \%$ glutaraldehyde, washed with PBS, and then fixed with $1 \%$ osmotetroxide. The tissues were dehydrated with acetone and sliced for later examination. After staining with $4 \%$ uranium acetate and $0.2 \%$ lead citrate, the ultrastructure of the tissue was observed by electron microscopy (JEM-1010; JEOL, Tokyo, Japan).

\section{Statistical analysis}

All data were statistically analysed by using SPSS 22.0 (IBM, Armonk, NY, USA). Continuous variables were expressed as mean $\pm S D$, and the difference between CCPR and ECPR groups was determined by the Student's $t$-test. Survival rates between the two groups were estimated by Kaplan-Meier analysis. $p<0.05$ was considered statistically significant.

\section{Results}

\section{Survival rate}

All swine were successfully induced into VF. Of the eight swine in the CCPR group, two died at 3.7 and 5.3 $\mathrm{h}$ after the ROSC, whereas the remaining survived for up to $6 \mathrm{~h}(6 / 8,67.5 \%)$. All eight animals in the ECPR group successfully survived to ROSC for $6 \mathrm{~h}(8 / 8,100 \%)$. The survival rate of the two groups was not statistically significant $(p>0.05)$ (Fig. 1). 


\section{Haemodynamics}

Table 1 summarises the HR and MAP of the CCPR and ECPR groups. They did not differ statistically at the baseline ( $p>0.05)$. The HR in ROSC and 1, 2, 4, $6 \mathrm{~h}$ after ROSC were significantly higher in the CCPR than in the ECPR group ( $p<0.01$ ) (Fig. 2a). The MAP in ROSC and 1, 2, $4 \mathrm{~h}$ after ROSC were significantly lower in the CCPR than in the ECPR group $(p<0.05)$, whereas differences in the ROCS at $6 \mathrm{~h}(p>0.05)$ were not (Fig. 2b).

Table 1. The heart rate and mean arterial pressure of the CCPR and ECPR groups

\begin{tabular}{|llll|}
\hline Variables & CCPR & ECPR & p-value \\
\hline HR (bpm) & & & \\
\hline Baseline & $118.25 \pm 7.59$ & $119.25 \pm 6.84$ & 0.768 \\
\hline ROSC & $161.25 \pm 12.63$ & $126.75 \pm 7.48$ & $<0.0001$ \\
\hline ROSC 1 h & $149.50 \pm 14.01$ & $120.25 \pm 12.02$ & 0.001 \\
\hline ROSC2 h & $144.00 \pm 13.35$ & $118.50 \pm 9.72$ & 0.001 \\
\hline ROSC 4 h & $132.29 \pm 11.22$ & $115.00 \pm 4.54$ & 0.006 \\
\hline ROCS 6 h & $132.67 \pm 5.89$ & $115.00 \pm 6.14$ & $<0.0001$ \\
\hline MAP $(\mathrm{mmHg})$ & & \\
\hline Baseline & $114.44 \pm 5.44$ & $115.55 \pm 3.61$ & 0.637 \\
\hline ROSC & $77.46 \pm 4.33$ & $104.61 \pm 10.89$ & $<0.0001$ \\
\hline ROSC1h & $82.44 \pm 7.09$ & $103.04 \pm 8.28$ & $<0.0001$ \\
\hline ROSC2h & $83.00 \pm 8.53$ & $105.44 \pm 8.12$ & $<0.0001$ \\
\hline ROSC4h & $96.80 \pm 6.07$ & $106.14 \pm 9.25$ & 0.037 \\
\hline ROCS6h & $99.55 \pm 11.00$ & $107.16 \pm 8.97$ & 0.178 \\
\hline
\end{tabular}

$\mathrm{HR}$, heart rate; MAP, mean arterial pressure; CCPR, conventional cardiopulmonary resuscitation; ECPR, extracorporeal cardiopulmonary resuscitation; ROSC, return of spontaneous circulation.

\section{Blood and tissue biomarkers}

There were no significant differences in serum SP-A, SP-D, CC16, MDA, and SOD at baseline between the CCPR and ECPR groups ( $p>0.05$ ). Serum SP-A, SP-D, CC16, MDA were found in ROSC and at 1, 2, 4, $6 \mathrm{~h}$ after ROSC were statistically higher in the CCPR than in the ECPR group $(p<0.05)$, whereas the serum SOD was lower at the abovementioned five-time points in the CCPR than in the ECPR group $(p<0.01)$ (Table 2 and Fig. 3a, 3b, 3c, 3d, 3e). The comparison of the tissues from the two groups showed that MDA and 
MPO were significantly higher in the CCPR than in the ECPR group, whereas the SP-A, SP-D, and SOD CCPR were significantly lower in the CCPR than in the ECPR group $(p<0.01)$ (Table 3 and Fig. $4 a, 4 b, 4 c$, $4 d, 4 e)$.

Table 2. Blood biomarkers of the CCPR and ECPR groups 


\begin{tabular}{|c|c|c|c|}
\hline Variables & CCPR & ECPR & $p$-value \\
\hline \multicolumn{4}{|c|}{ SP-A (ng/mL) } \\
\hline Baseline & $40.35 \pm 5.30$ & $40.97 \pm 5.57$ & 0.824 \\
\hline ROSC & $114.02 \pm 15.47$ & $86.88 \pm 9.42$ & 0.001 \\
\hline ROSC $1 \mathrm{~h}$ & $91.76 \pm 15.72$ & $64.24 \pm 8.46$ & 0.001 \\
\hline ROSC $2 \mathrm{~h}$ & $86.76 \pm 13.34$ & $60.10 \pm 6.77$ & $<0.0001$ \\
\hline ROSC $4 \mathrm{~h}$ & $78.33 \pm 18.29$ & $53.05 \pm 9.21$ & 0.004 \\
\hline ROCS $6 \mathrm{~h}$ & $76.59 \pm 17.94$ & $46.16 \pm 6.35$ & 0.007 \\
\hline \multicolumn{4}{|c|}{ SP-D (ng/mL) } \\
\hline Baseline & $67.12 \pm 7.09$ & $63.69 \pm 5.31$ & 0.292 \\
\hline ROSC & $179.72 \pm 28.94$ & $138.16 \pm 19.54$ & 0.005 \\
\hline ROSC $1 \mathrm{~h}$ & $142.23 \pm 35.60$ & $100.83 \pm 15.67$ & 0.009 \\
\hline ROSC 2 h & $132.57 \pm 33.37$ & $93.88 \pm 15.85$ & 0.010 \\
\hline ROSC $4 \mathrm{~h}$ & $126.17 \pm 37.79$ & $84.10 \pm 21.86$ & 0.019 \\
\hline ROCS $6 \mathrm{~h}$ & $131.67 \pm 30.73$ & $73.23 \pm 20.37$ & 0.001 \\
\hline \multicolumn{4}{|c|}{ CC16 (ng/mL) } \\
\hline Baseline & $147.26 \pm 24.91$ & $159.60 \pm 16.36$ & 0.261 \\
\hline ROSC & $457.39 \pm 75.75$ & $335.76 \pm 44.11$ & 0.002 \\
\hline ROSC $1 \mathrm{~h}$ & $365.60 \pm 65.90$ & $254.67 \pm 38.54$ & 0.001 \\
\hline ROSC $2 \mathrm{~h}$ & $338.92 \pm 60.38$ & $234.35 \pm 31.83$ & 0.001 \\
\hline ROSC $4 \mathrm{~h}$ & $315.87 \pm 61.34$ & $217.04 \pm 38.49$ & 0.002 \\
\hline ROCS $6 \mathrm{~h}$ & $308.20 \pm 62.09$ & $184.68 \pm 45.05$ & 0.001 \\
\hline \multicolumn{4}{|c|}{ MDA (nmol/mL) } \\
\hline Baseline & $4.00 \pm 0.66$ & $4.11 \pm 0.49$ & 0.708 \\
\hline ROSC & $13.37 \pm 1.44$ & $7.54 \pm 2.30$ & $<0.0001$ \\
\hline ROSC $1 \mathrm{~h}$ & $12.53 \pm 1.23$ & $6.29 \pm 1.10$ & $<0.0001$ \\
\hline ROSC 2 h & $12.73 \pm 1.06$ & $6.21 \pm 0.93$ & $<0.0001$ \\
\hline
\end{tabular}




\begin{tabular}{|llll|}
\hline ROSC 4 h & $13.23 \pm 1.19$ & $5.79 \pm 1.17$ & $<0.0001$ \\
\hline ROCS 6 h & $12.72 \pm 0.91$ & $5.05 \pm 0.63$ & $<0.0001$ \\
\hline SOD (U/mL) & & & \\
\hline Baseline & $219.19 \pm 15.69$ & $215.48 \pm 24.85$ & 0.727 \\
\hline ROSC & $141.85 \pm 18.71$ & $210.60 \pm 25.01$ & $<0.0001$ \\
\hline ROSC 1 h & $123.93 \pm 18.10$ & $198.30 \pm 26.71$ & $<0.0001$ \\
\hline ROSC 2 h & $113.01 \pm 18.64$ & $195.25 \pm 18.55$ & $<0.0001$ \\
\hline ROSC 4 h & $104.85 \pm 22.13$ & $203.04 \pm 16.68$ & $<0.0001$ \\
\hline ROCS 6 h & $105.83 \pm 26.29$ & $197.78 \pm 26.23$ & $<0.0001$ \\
\hline
\end{tabular}

CCPR, conventional cardiopulmonary resuscitation; ECPR, extracorporeal cardiopulmonary resuscitation; ROSC, return of spontaneous circulation; SP-A, pulmonary surfactant protein A; SP-D, pulmonary surfactant protein $D ; C C 16$, Clara cell protein 16; MDA, malondialdehyde; SOD, superoxide dismutase.

Table 3. Tissue biomarkers of the CCPR and ECPR groups

\begin{tabular}{|llll|}
\hline Variables & CCPR & ECPR & $p$-value \\
\hline SP-A $(\mathrm{ng} / \mathrm{mL})$ & $32.60 \pm 4.13$ & $71.87 \pm 18.88$ & $<0.0001$ \\
\hline SP-D $(\mathrm{ng} / \mathrm{mL})$ & $45.08 \pm 3.40$ & $131.29 \pm 22.80$ & $<0.0001$ \\
\hline MDA $(\mathrm{nmol} / \mathrm{mL})$ & $11.05 \pm 1.07$ & $5.67 \pm 1.62$ & $<0.0001$ \\
\hline SOD $(\mathrm{U} / \mathrm{mL})$ & $82.92 \pm 32.02$ & $158.65 \pm 20.68$ & $<0.0001$ \\
\hline MPO $(\mathrm{ng} / \mathrm{mL})$ & $634.66 \pm 54.62$ & $274.08 \pm 99.78$ & $<0.0001$ \\
\hline
\end{tabular}

CCPR, conventional cardiopulmonary resuscitation; ECPR, extracorporeal cardiopulmonary resuscitation; SP-A, pulmonary surfactant protein A; SP-D, pulmonary surfactant protein D; MDA, malondialdehyde; SOD, superoxide dismutase; MPO, myeloperoxidase.

\section{EVLW and PVPI results}

Table 4 compares the EVLW and PVPI of the CCPR and EPCR groups. At baseline, there was no significant difference between the two groups ( $p>0.05)$. The EVLW at ROSC6h in both groups was statistically different $(p<0.01$ ) (Fig. 5a). PVPI values at ROSC6h in the two groups showed significant differences $(p<0.01)$ (Fig. 5b). Moreover, the EVLW values at ROSC6h compared with the baseline in both two groups showed statistically significant differences $(p<0.05)$ (Fig. 5c, 5d). The PVPI in the CCPR group was statistically higher compared to the baseline $(p<0.01)$, whereas no difference was observed in ECPR group ( $p>0.05$ ) (Fig. 5e, 5f). 
Table 4. Comparison of the extravascular lung water and pulmonary vascular permeability index of the CCPR and ECPR groups

\begin{tabular}{|llll|}
\hline Variable & \multicolumn{1}{l}{ Baseline } & \multicolumn{1}{l}{ ROSC6h } & $p$-value \\
\hline EVLW $(\mathrm{mL} / \mathrm{kg})$ & & \\
\hline CCPR & $9.61 \pm 1.37$ & $21.85 \pm 3.92$ & $<0.0001$ \\
ECPR & $9.39 \pm 1.70$ & $11.78 \pm 1.82^{\mathrm{a}}$ & 0.017 \\
\hline PVPI & & & \\
\hline CCPR & $2.24 \pm 0.50$ & $5.97 \pm 1.39$ & 0.001 \\
\hline ECPR & $2.15 \pm 0.43$ & $2.06 \pm 0.91^{\mathrm{a}}$ & 0.812 \\
\hline
\end{tabular}

EVLW, extravascular lung water; PVPI, pulmonary vascular permeability index; CCPR, conventional cardiopulmonary resuscitation; ECPR, extracorporeal cardiopulmonary resuscitation; ROSC, return of spontaneous circulation. ${ }^{a} p<0.0001$ vs. CCPR group.

\section{Results from electron microscopy}

Electron microscopy revealed serious damage that was evident in the broadening of the blood-gas barrier in the CCPR group; in the epithelial cells, the type II lamellar bodies were empty vacuoles for the most part. In the ECPR group, the blood-gas barrier was clear, without any clear broadening, with epithelial cells showing non-empty type II lamellar bodies (Fig. 6a, 6b, 6c, 6d).

\section{Discussion}

This animal experiment showed that ECPR has a better pulmonary protective effect than CCPR. Compared with the ECPR group, the CCPR group had more severe oxidative stress injury and worse scavenging ability for oxygen-free radicals. In the ECPR group, more protective active proteins were present on the alveolar surface, the blood-gas barrier was intact, there was a greater abundance of the alveolar surface-active protein in the lamellar body and less pulmonary oedema.

The pulmonary surfactant protein is secreted and released from type II alveolar epithelial cells. It plays an important role in maintaining lung surface tension and participates in the body's defense functions. Pulmonary surface proteins are composed of four proteins: namely, hydrophobic small molecular proteins SP-B and SP-C, which are mainly involved in maintaining alveolar surface tension, and hydrophilic large molecular proteins SP-A and SP-D, which are mainly involved in immune and inflammatory mechanisms [16]. SP-A and SP-D can minimise lung injury by reducing the production of inflammatory factors and can clear various pathogens [17]. Experiments have shown that SP-A and SP-D play an important role in the innate immunity of the lungs and the prevention of infection [18]. Meanwhile, SP-A and SP-D have strong antioxidant functions, and these two proteins can alleviate and 
prevent the oxidative stress response of the lungs induced by various etiologies [19]. Furthermore, SP-A can control apoptosis and stabilise alveolar epithelial cells [20]. Meanwhile, the pulmonary clearance of exogenous substances can be enhanced by regulating inflammatory cells and promoting macrophage phagocytosis [17]. Low SP-A expression in the lung tissues will lead to decreased stability of alveolar epithelial cells and increase the possibility of pulmonary oedema, pulmonary infection, and pulmonary injury [21]. Lung injury will lead to the reduction of SP-A and SP-D in the lung tissues [17, 22]. Greater secretion of SP-D in these tissues has a clear protective effect on the lungs against infection, promotes macrophage metastasis, and maintains the stability of alveolar epithelial cells [23, 24]. Studies have suggested that the injury to the blood-gas barrier caused by lung injury, especially injury to alveolar epithelial cells, will lead to the increased secretion of serum SP-A and SP-D [25]. Serum SP-D concentration is positively correlated with the degree of lung injury [26] as well as mortality [27]. Clara cells have shown to repair damaged epithelial cells; detoxify the invasion of foreign organisms; and secrete SP-D, SP-A, CC16 protein, and other substances [28]. When Clara cells are damaged or there are changes in alveolar epithelial permeability, the function of synthesis and secretion of CC16 protein of Clara cells changes, which is of great value in the diagnosis of lung injury [26]. Increased serum concentrations of $\mathrm{CC} 16$ can clearly indicate the presence of lung injury and positively correlated with the degree of lung injury [29-31]. SOD is a key enzyme capable of scavenging oxygen free radicals, which protects cells from superoxide damage by catalytic oxidation of superoxide free radicals into molecular oxygen and hydrogen peroxide, thereby inhibiting oxidative protein modification and lipid peroxidation in the cell membrane [32]. In addition, SOD can increase the bioavailability of nitric oxide (NO) by competitively combining with superoxide to protect tissues and organs [33]. Other studies have shown that SOD can reduce lung injury caused by hyperventilation [34]. MDA is the final product of the lipid peroxidation of the main structure of the cell membrane and occurs because of the degradation of polyunsaturated lipids. Lipid peroxidation is a mature mechanism of cell damage in plants and animals, and it is used as an indicator of oxidative stress in cells and tissues [35]. MDA is a terminal product produced by cell damage or free radicals and can be used as an indicator of lung injury [36]. MPO is an important enzyme released by neutrophils and promotes the formation of hypo chloric acid, a powerful oxidant associated with bactericidal effects and tissue destruction through induction of necrosis and apoptosis [37, 38]. In previous studies, increased tissue MPO has been associated with aggravated oxidative damage of tissues $[34,39,40]$. Oxidative damage leads to the destruction of the lipid structure of tissue cells, and the subsequent increase of MDA in tissues [34,41,42]. This process will excessively consume SOD, resulting in a sharp decline in SOD levels [34,42]. However, ischaemia-reperfusion injury will produce a large amount of oxygen free radicals [43]. Several experiments have shown that, in addition to histological examination, serum examination of MDA and SOD can reflect the degree of oxidative damage to tissues $[39,41,44]$.

The results of this study showed that serum MDA in the CCPR group was significantly higher than in the ECPR group. Moreover, the serum MDA level in the CCPR group was relatively stable at $6 \mathrm{~h}$ after ROSC, although the serum MDA in the ECPR group showed a slowly decreasing trend within $6 \mathrm{~h}$. This indicates that the oxygen free radicals that are not completely cleared in the lungs and persisted in the CCPR group 
after ROSC, resulting in the sustained destruction of lipids. However, the continuous decrease of serum MDA in the ECPR group may be related to the mild oxidative free radical damage and lower MDA production rate than indicative of an association with the scavenging rate. Serum SOD in the CCPR group showed a continuous declining trend, although the change of serum SOD in the ECPR group was not obvious. Furthermore, serum SOD in the ECPR group did not change significantly compared with the baseline value, and the degree of decrease was limited. In the CCPR group, SOD was involved in oxygenfree radical scavenging in tissues, which results in the continuous consumption of SOD. The oxidative radical damage in the ECPR group was mild, which slowed down the consumption of SOD. This experiment proved that the CCPR group could sustain more oxidative free radicals at the beginning of ROSC than the ECPR group and will bear greater oxidative damage after ROSC. The serum SP-A, SP-D, and CC16 levels in the two groups showed a decreasing trend following a significant increase after ROSC, and the values in the CCPR group was significantly higher than in the ECPR group, indicating that the lung injury was more serious in the CCPR than in the ECPR group. Lung injury was severe in both groups immediately after successful resuscitation and gradually decreased thereafter. Moreover, lung SP-A and SP-D in the CCPR group were significantly lower than those in the ECPR group, indicating that the lung injury in the CCPR group was more serious than that in the ECPR group.

As can be seen from the EVLW and PVPI results measured by the thermal dilution method, pulmonary oedema in the CCPR group was more serious when comparing the changes of EVLW and baseline values of the experimental animals in the CCPR group and the ECPR group at ROSC6h. The PVPI value of the CCPR group showed an abnormal increase at ROSC6h, whereas the PVPI value of ECPR group remained in the normal range and did not differ from the baseline PVPI value. The increase in PVPI is attributable to permeable pulmonary oedema; therefore, pulmonary oedema due to excess volume can be excluded. In comparison, we found that the increased EVLW in the CCPR group was mainly caused by permeable pulmonary oedema. The serum and tissue SP-A and SP-D showed that the loss of SP-A and SP-D was serious in the CCPR group because of the release of more surface-active material through damage to the blood-gas barrier rather than by secretion into the alveoli, which subsequently led to changes in the lung microstructure that increased alveolar tension, abnormal changes of permeability, and contributed to the severity of the permeable pulmonary oedema. Through this between-group comparison, we found that ECMO has a positive significance in reducing pulmonary fluid extravasation and can stabilise the vascular permeability of the lungs. Moreover, it indicated that the ECPR group had significantly lower pulmonary oedema compared with the CCPR group, indicating the lung function protection.

Furthermore, histology on electron microscopic examination showed that the CCPR group had severe alveolar type II lamellar body cell loss and inadequate storage of alveolar surface-active protein, which increased the damage to the blood-gas barrier, indicating an inability to guarantee the stability of the alveolar membrane permeability and increased permeability leading to pulmonary oedema. However, the ECPR group retained better-organised microstructure, showed alveolar type II cells with plenty of stored alveolar surface-active materials, which can be secreted as needed into the alveolar space. There was no serious damage to the blood-gas barrier, which maintains the optimal functioning of the barrier. Based on the histologic changes between the two groups, it is reasonable to assume that the ECPR group could 
show greater resistance to resuscitated pulmonary exogenous infection than the CCPR group when the duration of treatment is prolonged.

The limitations of our experimental study were as follows. Firstly, it was nearly impossible to perform large-scale animal experiments; therefore, the sample size was small and there was a high possibility of error and bias. Meanwhile, to enable ECMO to be added into the previous treatment process of ROSC, the catheterisation for ECMO was completed well in advance in this experiment. However, the timely placement and operation of ECMO are extremely difficult to be complete quickly in practical circumstances. Therefore, although this study concluded that the early combined application of ECMO had a positive effect, there remained some obstacles to achieve better promotion and development of this technology. Meanwhile, due to the addition of ECMO in the ECPR group, we set the thermostat of the ECMO machine to $34^{\circ} \mathrm{C}$, which could have implied a mild temperature effect on the ECPR group. However, the results in the literature suggest that hypothermia has no significant benefit for CA resuscitation [45]. Therefore, we did not undertake an in-depth investigation into the effect of the abovementioned hypothermia.

\section{Conclusion}

Early application of ECPR has a better protective effect on post-resuscitation lung injury than CCPR. Furthermore, ECPR has a protective effect on the post-resuscitation pulmonary injury, and its mechanism may be partly related to the regulation of alveolar surface-active protein and the improvement of oxidative stress response after resuscitation.

\section{Abbreviations}

CA, cardiac arrest; CC16, Clara cell protein 16; CCPR, conventional cardiopulmonary resuscitation; CPR, cardiopulmonary resuscitation; ECMO, extracorporeal membrane oxygenation; ECPR, extracorporeal cardiopulmonary resuscitation; ELISA, enzyme-linked immunosorbent assay; EVLW, extravascular lung water; HR, monitor the heart rate; MAP, mean arterial pressure; MDA, malondialdehyde; MPO, myeloperoxidase; NO, nitric oxide; PBS, phosphate-buffered saline; PVPI, pulmonary vascular permeability index; ROSC, return of spontaneous circulation; SOD, superoxide dismutase; SP-A, surfactant protein A; SP-D, surfactant protein D.

\section{Declarations}

\section{Ethical approval}

This study was approved by the Capital Medical University Institutional Animal Care Committee and the Beijing Chao-Yang Hospital Affiliated to the Capital Medical University Animal Care and Use Committee.

\section{Consent for publication}


Not applicable

\section{Availability of data and materials}

The datasets used and/or analysed during the current study are available from the corresponding author on reasonable request.

\section{Competing interests}

The authors declare they have no conflicts of interest regarding this article.

\section{Funding}

None.

\section{Author contributions}

Chun-Sheng Li conceived this study, designed the trial and obtained research funding. Ji-Yang Ling, Yun Zhang, Xiao-Li Yuan, Bo Liu, Yong Liang and Qiang Zhang conducted the trial and collected data. JiYang Ling analysed data, performed statistical analysis, drafted and revised the manuscript. ChunSheng Li takes responsibility for the paper as a whole. All authors read and approved the final manuscript.

\section{Acknowledgments}

We thank Lian-Xing Zhao for his technical assistance for this research. We thank the Beijing Key Laboratory of Cardiopulmonary Cerebral Resuscitation, Beijing Chao-Yang Hospital, Capital Medical University, Beijing, People's Republic of China for providing the laboratory. We would like to thank Editage (www.editage.com) for English language editing.

\section{References}

1. Lin MH, Peng LN, Chen LK, Chen TJ, Hwang SJ. Cardiopulmonary resuscitation for hospital inpatients in Taiwan: an 8-year nationwide survey. Resuscitation. 2012;83:343-6.

2. Goldberg SA, Metzger JC, Pepe PE. Year in review 2011: Critical care-out-of-hospital cardiac arrest and trauma. Crit Care. 2012;16:247.

3. Nichol G, Thomas E, Callaway CW, Hedges J, Powell JL, Aufderheide TP, et al. Resuscitation outcomes consortium investigators: regional variation in out-of-hospital cardiac arrest incidence and outcome. JAMA. 2008;300:1423-31.

4. Nehme Z, Andrew E, Bernard S, Smith K. Impact of cardiopulmonary resuscitation duration on survival from paramedic witnessed out-of-hospital cardiac arrests: An observational study. Resuscitation. 2016;100:25-31. 
5. Chen YS, Chao A, Yu HY, Ko WJ, Wu IH, Chen RJ, et al. Analysis and results of prolonged resuscitation in cardiac arrest patients rescued by extracorporeal membrane oxygenation. J Am Coll Cardiol. 2003;41:197-203.

6. Chen YS, Lin JW, Yu HY, Ko WJ, Jerng JS, Chang WT, et al. Cardiopulmonary resuscitation with assisted extracorporeal life-support versus conventional cardiopulmonary resuscitation in adults with in-hospital cardiac arrest: an observational study and propensity analysis. Lancet. 2008;372:554-61.

7. Ortega-Deballon I, Hornby L, Shemie SD, Bhanji F, Guadagno E. Extracorporeal resuscitation for refractory out-of-hospital cardiac arrest in adults: A systematic review of international practices and outcomes. Resuscitation. 2016;101:12-20.

8. Kim SJ, Kim HJ, Lee HY, Ahn HS, Lee SW. Comparing extracorporeal cardiopulmonary resuscitation with conventional cardiopulmonary resuscitation: A meta-analysis. Resuscitation. 2016;103:106-16.

9. Thiagarajan RR, Laussen PC, Rycus PT, Bartlett RH, Bratton SL. Extracorporeal membrane oxygenation to aid cardiopulmonary resuscitation in infants and children. Circulation. 2007;116:1693-700.

10. Stub D, Byrne M, Pellegrino V, Kaye DM. Extracorporeal membrane oxygenation to support cardiopulmonary resuscitation in a sheep model of refractory ischaemic cardiac arrest. Heart Lung Circ. 2013;22:421-27.

11. Menegazzi JJ, Salcido DD, Housler GJ, Logue ES. Feasibility of initiating extracorporeal life support during mechanical chest compression CPR: a porcine pilot study. Resuscitation. 2012;83:130-3.

12. Stub D, Bernard S, Pellegrino V, Smith K, Walker T, Sheldrake J, et al. Refractory cardiac arrest treated with mechanical CPR, hypothermia, ECMO and early reperfusion (the CHEER trial). Resuscitation. 2015;86:88-94.

13. Kleinman ME, Brennan EE, Goldberger ZD, Swor RA, Terry M, Bobrow BJ, et al. Adult basic life support and cardiopulmonary resuscitation quality 2015 American Heart Association Guidelines update for cardiopulmonary resuscitation and emergency cardiovascular care. Circulation. 2015;132:414-S35.

14. Link MS, Berkow LC, Kudenchuk PJ, Halperin HR, Hess EP, Moitra VK, et al. Part 7: Adult advanced cardiovascular life support 2015 American Heart Association Guidelines update for cardiopulmonary resuscitation and emergency cardiovascular care. Circulation. 2015;132:444-S64.

15. Callaway CW, Donnino MW, Fink EL, Geocadin RG, Golan E, Kern KB, et al. Part 8: Post-cardiac arrest care 2015 American Heart Association Guidelines update for cardiopulmonary resuscitation and emergency cardiovascular care. Circulation. 2015;132:465-S82.

16. Wright JR. Immunoregulatory functions of surfactant proteins. Nat Rev Immunol. 2005;5:58-68.

17. Sawa GE, Allen T, Wiener-Kronish L, Hawgood J. S. Surfactant proteins A and D enhance pulmonary clearance of Pseudomonas aeruginosa. Am J Respir Cell Mol Biol. 2006;34:704-10.

18. Crouch E1. Wright JR. Surfactant proteins A and D and pulmonary host defense. Rev Physiol. 2001;63:521-54. 
19. Bridges JP, Davis HW, Damodarasamy M, Kuroki Y, Howles G, Hui DY, et al. Pulmonary surfactant proteins $A$ and $D$ are potent endogenous inhibitors of lipid peroxidation and oxidative cellular injury. J Biol Chem. 2000;275:38848-55.

20. Ledford GH, Mukherjee JG, Noble S, Williams PW, Wright KL. JR. The role of surfactant protein A in bleomycin-induced acute lung injury. Am J Respir Crit Care Med. 2010;181:1336-44.

21. Boven BA, Dewachter C, Ruiz Patino L, Sokolow M, Rondelet Y. B. Influence of donor lung surfactant$a$ and -b protein expression on the development of primary graft dysfunction after lung transplantation: a pilot study. Ann Transplant. 2017;22:361-69.

22. Moran TS, Lionakis A, Mastrangelo M, Halder MA, del Pilar Huby T. M, et al. Restoration of lung surfactant protein D by IL-6 protects against secondary pneumonia following hemorrhagic shock. J Infect. 2014;68:231-41.

23. King BA, Kingma PS. Surfactant protein D deficiency increases lung injury during endotoxemia. Am J Respir Cell Mol Biol. 2011;44:709-15.

24. Sato A, Whitsett JA, Scheule RK, Ikegami M. Surfactant protein-d inhibits lung inflammation caused by ventilation in premature newborn lambs. Am J Respir Crit Care Med. 2010;181:1098-105.

25. Duda I, Grzybowska K, Jędrzejowska-Szypułka H, Lewin-Kowalik J. The sitting position during neurosurgical procedures does not influence serum biomarkers of pulmonary parenchymal injury. BMC Surg. 2012;12:24.

26. Turut KA, Acipayam H, Kirbas A, Yuce A, Cumhur Cure S. M, et al. Investigation of surfactant protein$D$ and interleukin- 6 levels in patients with blunt chest trauma with multiple rib fractures and pulmonary contusions: a cross-sectional study in Black Sea Region of Turkey. BMJ Open. 2016;6:e011797.

27. Ware LB, Koyama T, Billheimer DD, Wu W, Bernard GR, Thompson BT, et al. NHLBI ARDS Clinical Trials Network: Prognostic and pathogenetic value of combining clinical and biochemical indices in patients with acute lung injury. Chest. 2010;137:288-96.

28. Broeckaert F, Clippe A, Knoops B, Hermans C, Bernard A. Clara cell secretory protein (CC16): features as a peripheral lung biomarker. Ann N Y Acad Sci. 2000;923:68-77.

29. Wen MN, Zhao G, Zhang JY, Zhao YH. Clinical study on the changes of lung-specific proteins: CC16 after lung contusion. Exp Ther Med. 2017;14:2733-36.

30. Wutzler S, Lehnert T, Laurer H, Lehnert M, Becker M, Henrich D, et al. Circulating levels of Clara cell protein 16 but not surfactant protein D identify and quantify lung damage in patients with multiple injuries. J Trauma. 2011;71:E31-6.

31. Determann RM, Millo JL, Waddy S, Lutter R, Garrard CS, Schultz MJ. Plasma CC16 levels are associated with development of ALI/ARDS in patients with ventilator-associated pneumonia: a retrospective observational study. BMC Pulm Med. 2009;9:49.

32. Marikovsky M, Ziv V, Nevo N, Harris-Cerruti C, Mahler O. Cu/Zn superoxide dismutase plays important role in immune response. J Immunol. 2003;170:2993-3001. 
33. Jung O, Marklund SL, Geiger H, Pedrazzini T, Busse R, Brandes RP. Extracellular superoxide dismutase is a major determinant of nitric oxide bioavailability: in vivo and ex vivo evidence from ecSOD-deficient mice. Circ Res. 2003;93:622-9.

34. Lv H, Liu Q, Wen Z, Feng H, Deng X. Ci X. Xanthohumol ameliorates lipopolysaccharide (LPS)-induced acute lung injury via induction of AMPK/GSK3ß-Nrf2 signal axis. Redox Biol. 2017;12:311-24.

35. Karaiskos T, Tomos P, Asouhidou I, Nikiteas N, Kontakiotis T, Papalois A. Oxidative and preinflammatory stress in wedge resection of pulmonary parenchyma using the radiofrequency ablation technique in a swine model. J Cardiothorac Surg. 2012;7:7.

36. Esterbauer $\mathrm{H}$, Schaur RJ, Zollner $\mathrm{H}$. Chemistry and biochemistry of 4-hydroxynonenal, malonaldehyde and related aldehydes. Free Radic Biol Med. 1991;11:81-128.

37. Klebanoff SJ. Myeloperoxidase: friend and foe. J Leukoc Biol. 2005;77:598-625.

38. Kebir E, József D, Pan L, Wang W, Petasis L, Serhan NA. CN, et al. 15-epi-lipoxin A4 inhibits myeloperoxidase signaling and enhances resolution of acute lung injury. Am J Respir Crit Care Med. 2009;180:311-9.

39. Li ML, Lu L, Li S, Su K, Wang Z. Y, et al. The protective effect of dexmedetomidine on LPS-induced acute lung injury through the HMGB1-mediated TLR4/NF-kappaB and PI3K/Akt/mTOR pathways. Mol Immunol. 2018;94:7-17.

40. Schmalstieg FC, Keeney SE, Rudloff HE, Palkowetz KH, Cevallos M, Zhou X, et al. Arteriovenous CO2 removal improves survival compared to high frequency percussive and low tidal volume ventilation in a smoke/burn sheep acute respiratory distress syndrome model. Ann Surg. 2007;246:512-23.

41. Sener G, Paskaloglu K, Toklu H, Kapucu C, Ayanoglu-Dulger G, Kacmaz A, et al. Melatonin ameliorates chronic renal failure-induced oxidative organ damage in rats. J Pineal Res. 2004;36:232-41.

42. Zhang, Duan HX, Wang GL, Zhang CN, Zhu YQ, Liu XY. YJ. Protective effect of resveratrol against endotoxemia-induced lung injury involves the reduction of oxidative/nitrative stress. Pulm Pharmacol Ther. 2014;27:150-5.

43. Klausner JM, Paterson IS, Mannick JA, Valeri R, Shepro D, Hechtman HB. Reperfusion pulmonary edema. JAMA. 1989;261:1030-35.

44. Torun AC, Tutuncu S, Ustun B, Akdemir HU. A study of the therapeutic effects of resveratrol on blunt chest trauma-induced acute lung injury in rats and the potential role of endocan as a biomarker of inflammation. Inflammation. 2017;40:1803-10.

45. Nielsen N, Wetterslev J, Cronberg T, Erlinge D, Gasche Y, Hassager C, et al. TTM Trial Investigators: Targeted temperature management at 33 degrees $\mathrm{C}$ versus 36 degrees $\mathrm{C}$ after cardiac arrest. $\mathrm{N}$ Engl J Med. 2013;369:2197-206.

\section{Figures}




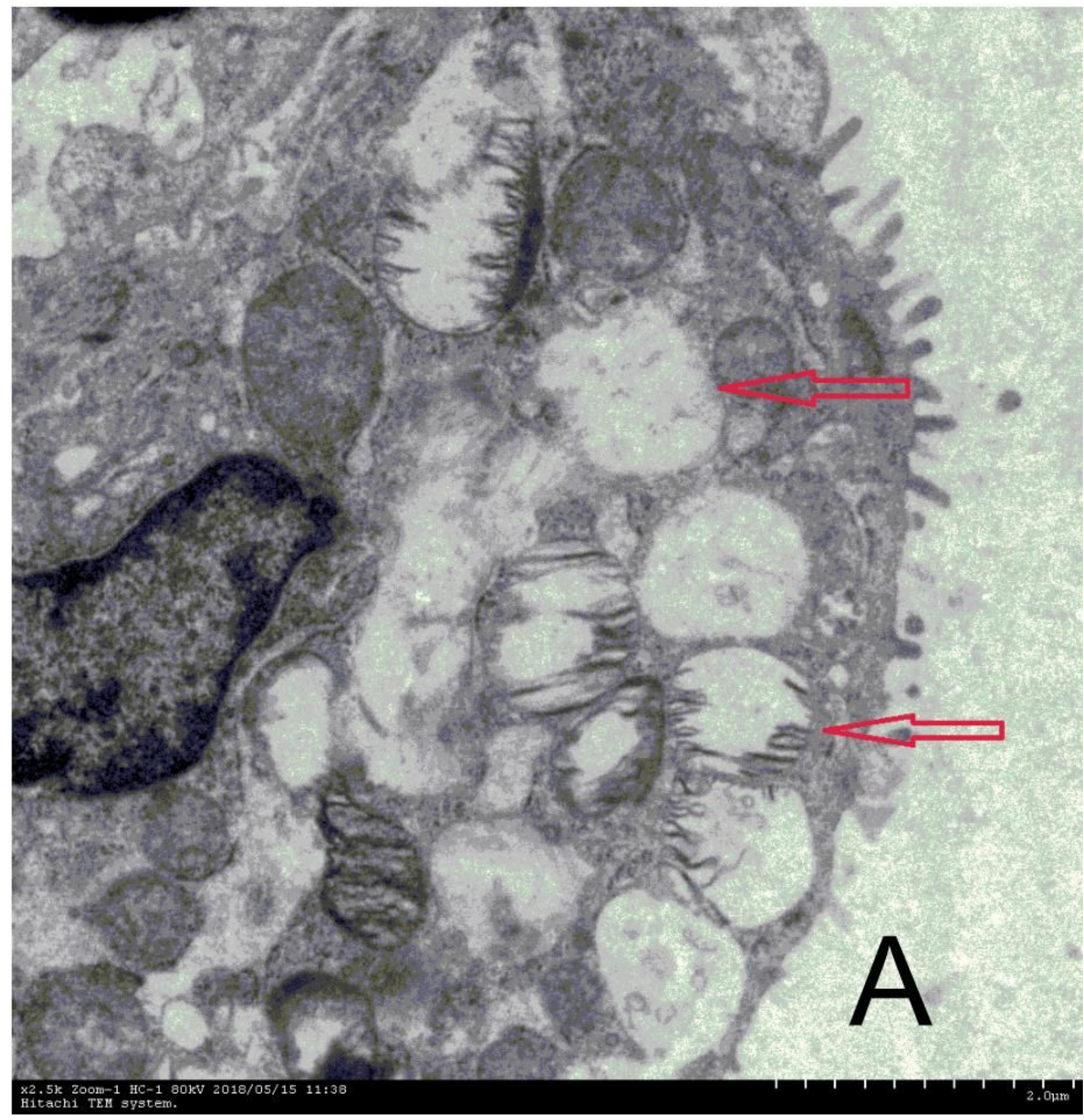

Figure 1

Organisational structure on electron microscopy (a) The arrow shows that, in the CCPR group, intracellular alveolar type II lamellar bodies are mostly vacuolated and have a fuzzy lamellar structure ( $\times 2.5 \mathrm{k}$ ) within the body. (b) In the ECPR group, the intracellular alveolar type II lamellar body appears complete and capable of releasing pulmonary surfactant protein ( $\times 2.5 \mathrm{k}$ ). (c) An arrow indicates the 
widening and blurring of the blood-gas barrier in the CCPR group ( $\times 12.0 \mathrm{k})$. (d) An arrow indicates that the structure of the blood-gas barrier is intact $(\times 12.0 \mathrm{k})$.

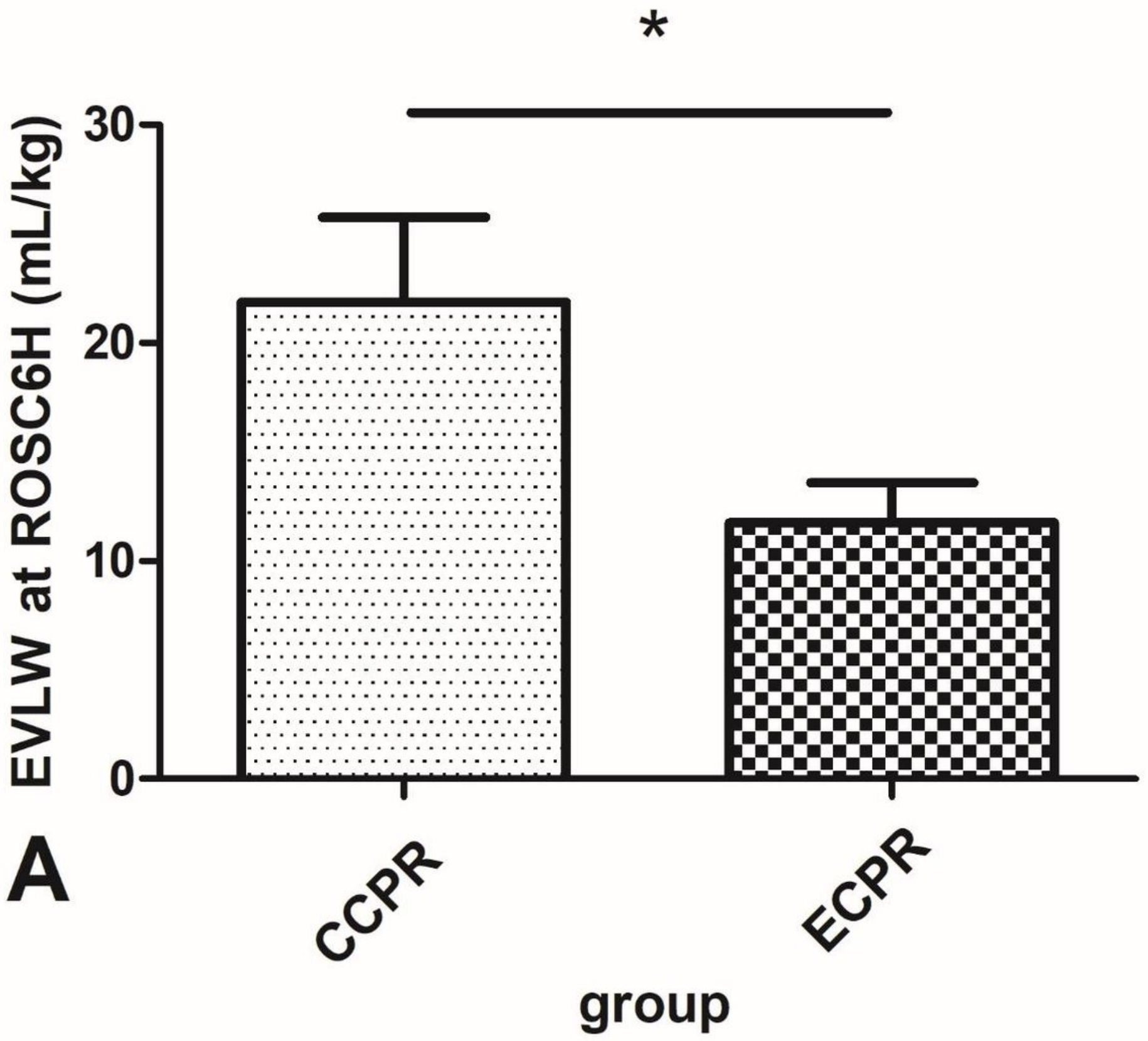

Figure 2

Pulmonary extravascular lung water and lung permeability index comparison between the CCPR and ECPR groups * $<0.01$ (a) The baseline for both groups of EVLW. (b) The increase of ROSC6h compared with the baseline value. (c) PVPI in the CCPR group. (d) PVPI in the ECPR group. EVLW, extravascular lung water; PVPI, pulmonary vascular permeability index; CCPR, conventional cardiopulmonary resuscitation; ECPR, extracorporeal cardiopulmonary resuscitation; ROSC, return of spontaneous circulation. 


\section{*}

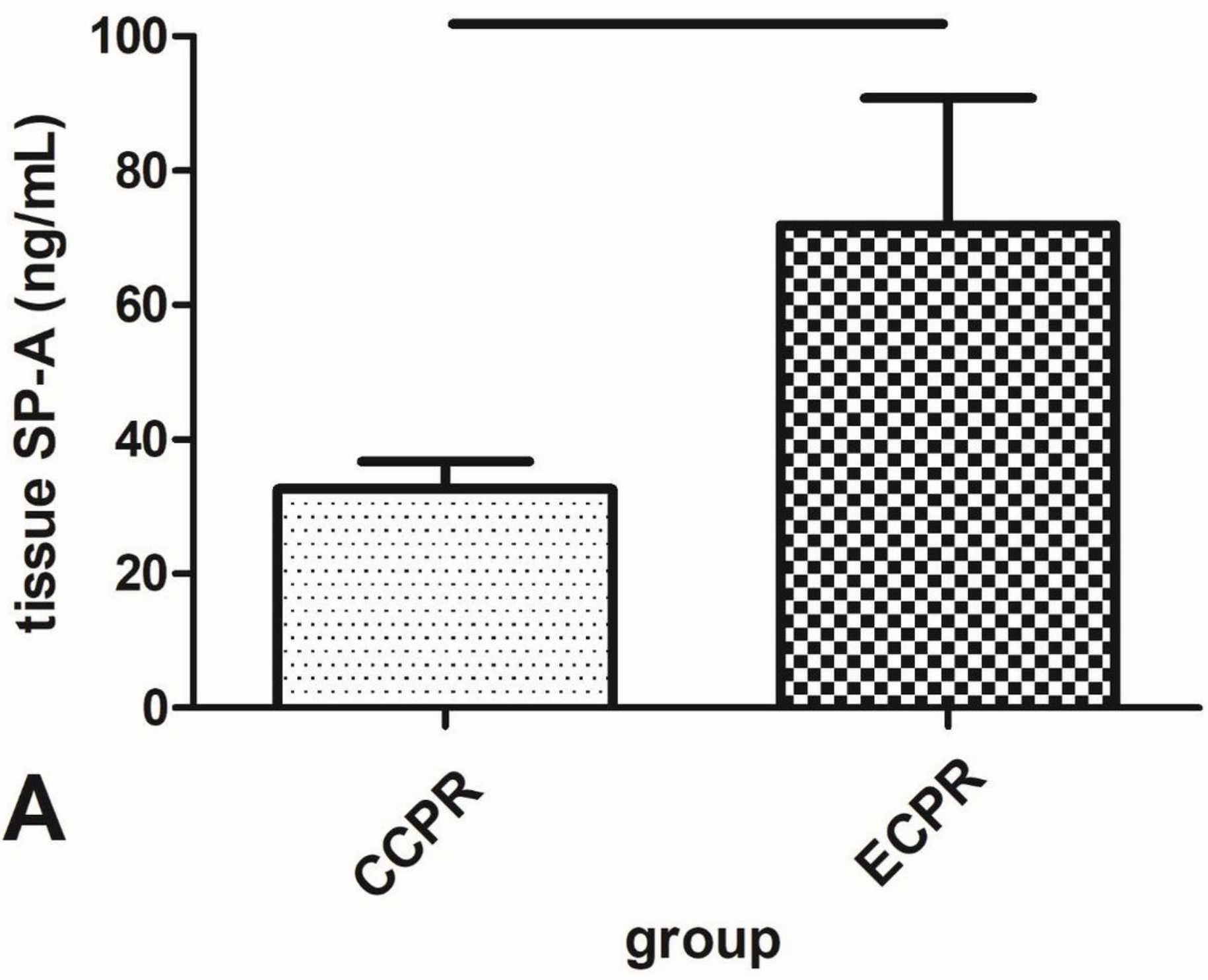

Figure 3

Comparison of biomarkers in lung histology between the CCPR and ECPR groups * $p<0.01$ CCPR, conventional cardiopulmonary resuscitation; ECPR, extracorporeal cardiopulmonary resuscitation. 


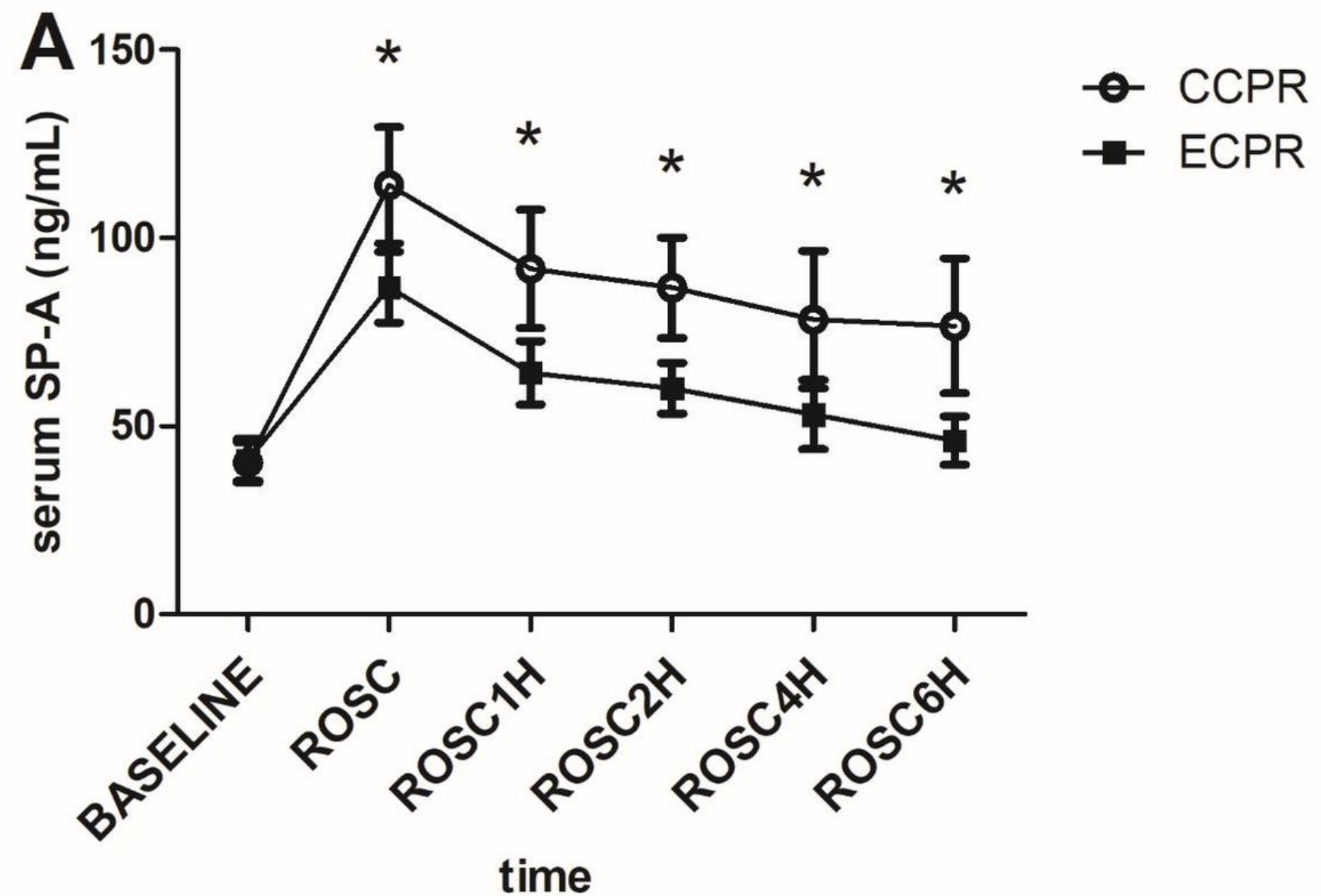

Figure 4

Comparison of serum markers at each time point between the CCPR and ECPR groups ${ }^{*} p<0.01$, and $\star \star p<0.05$. CCPR, conventional cardiopulmonary resuscitation; ECPR, extracorporeal cardiopulmonary resuscitation. 


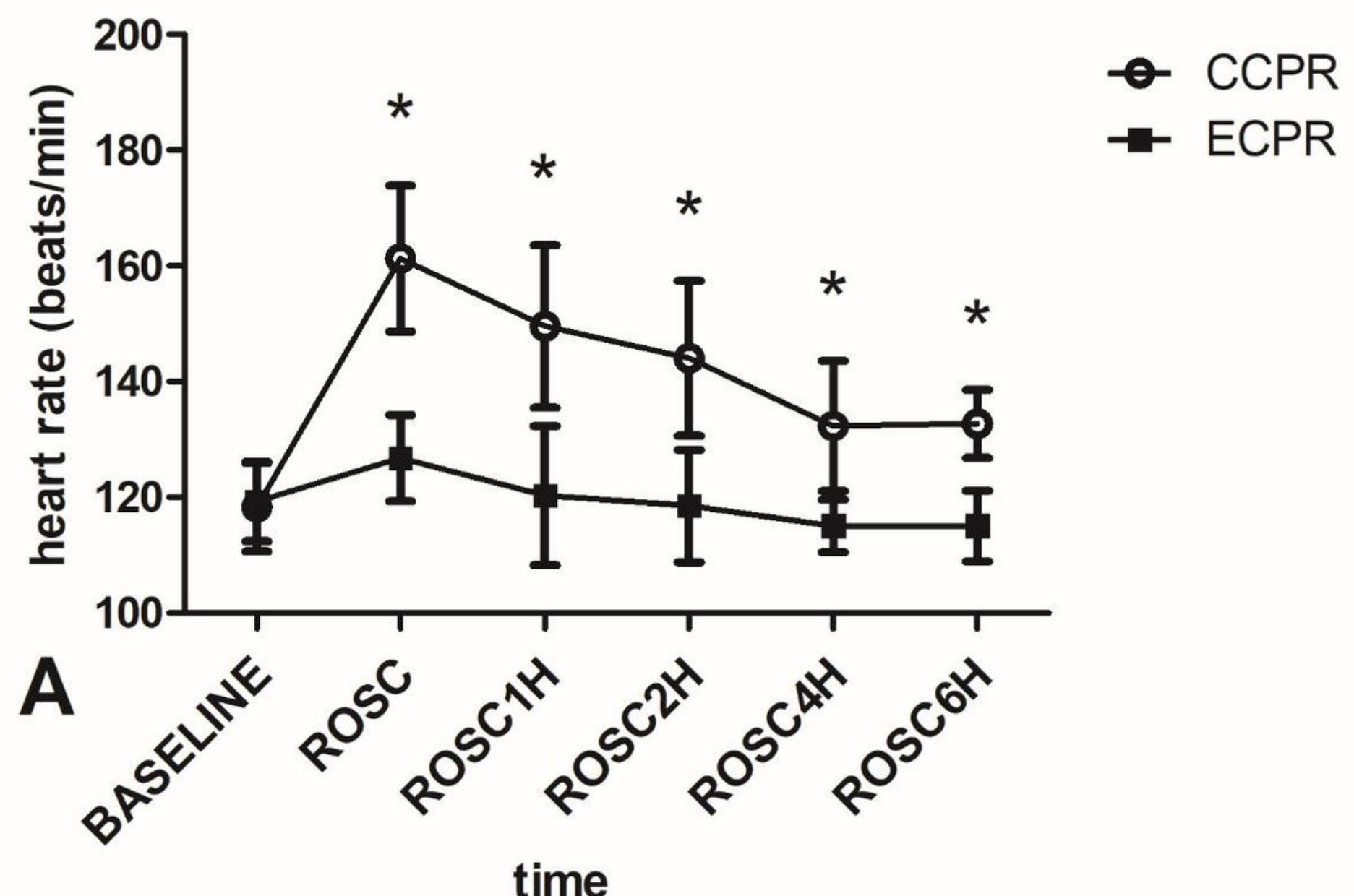

Figure 5

Comparison of HR and MAP at each time point between the CCPR and ECPR groups ${ }^{*} p<0.01,{ }^{*} p<0.05$. $\mathrm{HR}$, monitor the heart rate; MAP, mean arterial pressure; CCPR, conventional cardiopulmonary resuscitation; ECPR, extracorporeal cardiopulmonary resuscitation. 


\section{Survival of Data 1:Survival proportions}

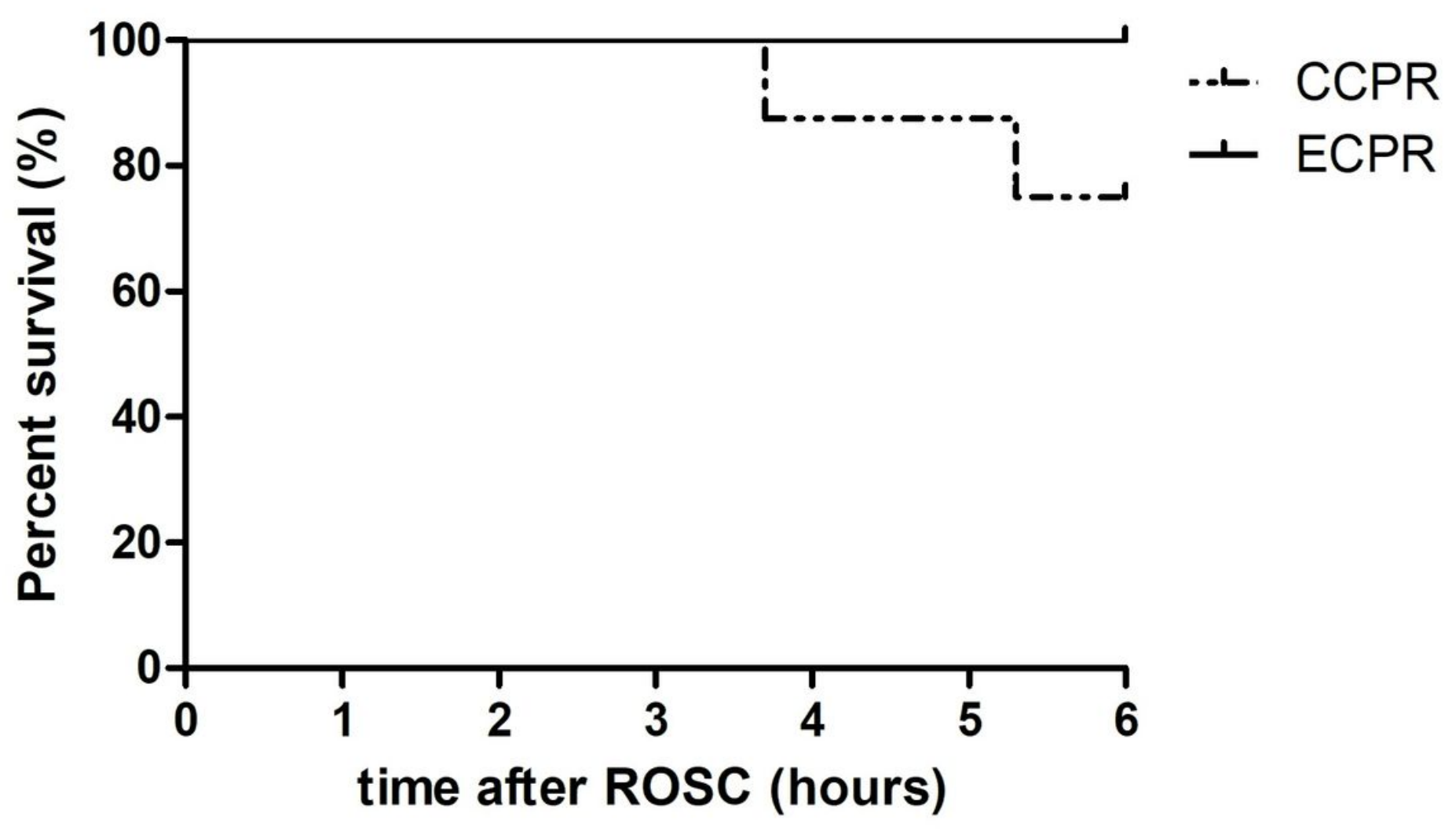

Figure 6

Comparison of the survival rate between the CCPR and ECPR groups CCPR, conventional cardiopulmonary resuscitation; ECPR, extracorporeal cardiopulmonary resuscitation.

\section{Supplementary Files}

This is a list of supplementary files associated with this preprint. Click to download.

- coveringletter.doc 